\title{
Outcomes of Conventional Trabeculectomy in Terms of Intraocular Pressure and Visual Acuity in Primary Open Angle Glaucoma
}

\author{
Erum Shahid ${ }^{1}$, Uzma Fasih ${ }^{2}$, Arshad Shaikh ${ }^{3}$ \\ ${ }^{1-3}$ Department of Ophthalmology, Abbasi Shaheed Hospital \& Karachi Medical and Dental College, Karachi
}

\begin{abstract}
Purpose: To determine the outcomes of conventional trabeculectomy in terms of decrease in intraocular pressure (IOP) and improvement in visual acuity in patients of primary open glaucoma, coming to a tertiary care hospital.
\end{abstract}

Study Design: Retrospective case series.

Place and Duration of Study: Department of Ophthalmology Abbasi Shaheed hospital from January 2017 to December 2018.

Methods: Patients undergoing trabeculectomy for Primary open angle glaucoma were included. Patients with repeated trabeculectomy, failed argon laser trabeculoplasty, advanced cataract, corneal opacities and absolute glaucoma were excluded. All the trabeculectomies were done under retrobulbar anaesthesia after taking all aseptic measures. Primary outcome measures were preoperative and postoperative visual acuity, intraocular pressure, number of anti-glaucoma medications and failed or successful trabeculectomy.

Results: Total 52 patients underwent trabeculectomy. There were $36(69.2 \%)$ males and $16(30.8 \%)$ females. Mean age was 56.73 years \pm 10.9 SD. Mean preoperative IOP was $30.96 \pm 6.71 \mathrm{~mm} \mathrm{Hg}$, post operatively IOP at 1 year was $15.6 \pm 4.2 \mathrm{~mm} \mathrm{Hg}$. P value was <.000. Mean number of antiglaucoma medications was 3.03 and 1.19 , pre and post-operatively respectively ( $p$ value $<.000$ ). Improvement in visual acuity after trabeculectomy was seen in $32(61.5 \%)$ patients. Complete Surgical success was seen in $28(53.8 \%)$ and qualified success in $21(40.4 \%)$ patients. Failed trabeculectomy was seen in 3 (5.8\%) patients.

Conclusion: Conventional trabeculectomy is effective in lowering IOP in primary open angle glaucoma patients. It maintains IOP within normal range with and without anti-glaucoma medications at 1 year follow-up. Trabeculectomy significantly reduces number of anti-glaucoma medications.

Key Words: Intraocular pressure, Primary open angle glaucoma, Trabeculectomy.

How to Cite this Article: Shahid E, Fasih U, Shaikh A. Outcomes of Conventional Trabeculectomy in Terms of Intraocular Pressure and Visual Acuity in Primary Open Angle Glaucoma. Pak J Ophthalmol. 2020, 36 (4): 386390.

Doi: https://doi.org/10.36351/pjo.v36i4.1086

Correspondence: Erum Shahid

Department of Ophthalmology

Abbasi Shaheed Hospital \& Karachi Medical and Dental College, Karachi

Email:drerum007@hotmail.com

Received: June 23, 2020

Accepted: August 8, 2020

\section{INTRODUCTION}

There are approximately 60 million people affected by glaucoma globally. It is the second leading cause of blindness after cataract. ${ }^{1}$ It is responsible for $10 \%$ of the total world blindness. ${ }^{2}$ Approximately half of the population with glaucoma resides in Asia including most populated areas including China, India and Pakistan. ${ }^{3}$ Fifty percent of the glaucoma patients are 
unaware of their disease at the time of diagnosis and they present at an advanced stage. ${ }^{4}$ Pakistan has burden of more than 1.8 million glaucoma patients and nearly half of them have lost their sight irreversibly due to delay in diagnosis and treatment. More and more people are becoming permanently blind in Pakistan due to untreated glaucoma and $90 \%$ of our population is ignorant of the disease. ${ }^{5}$

Trabeculectomy is the gold standard procedure for lowering intra ocular pressure (IOP) in primary open angle glaucoma (POAG) and normal tension glaucoma patients, not responding to medical and laser treatments. ${ }^{6}$ Studies comparing the results of initial treatment with trabeculectomy versus medical treatment reported superior results of trabeculectomy in lowering IOP. ${ }^{7}$ It has an additional benefit of stabilizing IOP by reducing diurnal fluctuation and dependence on patient compliance with medication. ${ }^{8}$ Most of the trabeculectomies attain successful outcome in reducing IOP over long term and thereby minimizing progression of glaucoma. ${ }^{6,9}$ Trabeculectomy has been modified with use of antimetabolites, limbal versus fornix based flaps and argon suture lysis. Early postoperative wound leak is a risk factor for failed trabeculectomy reported by 5 Flourouracil filtration surgical study. ${ }^{10}$

Surgical failure is the most frequent complication of trabeculectomy that leads to high postoperative IOP, progression of visual field loss and eventual blindness. ${ }^{8}$ Most frequent cause of failure is scar formation at conjunctiva-tenons-episcleral interface and at sclerostomy site. ${ }^{11,12}$

Poor compliance, multi drug therapy, lost to follow-up, financial burden are major concerns of glaucoma patients especially those coming to public sector hospitals of a developing country. Trabeculectomy in these circumstances would be beneficial for such patients.

This purpose of this study was to document the outcomes of trabeculectomy in terms of IOP and visual acuity in our population. This study will help in generating a local database in this context.

\section{METHODS}

A retrospective case series was conducted in the Department of Ophthalmology, Abbasi Shaheed hospital from January 2017 to Dec 2018. The study adheres to the tenants of declaration of Helsinki. We included patients who underwent trabeculectomy for
Primary open angle glaucoma. Patients with advanced cataract, corneal opacities, absolute glaucoma, history of failed trabeculectomy and failed argon laser trabeculoplasty were excluded from the study. Data was retrieved and collected from the hospital records about age, gender, duration and type of glaucoma, laterality of eye, preoperative and postoperative IOP, visual acuity and number of medications used. All the trabeculectomies were done under retrobulbar anaesthesia after taking all aseptic measures.

All the surgeries had fornix based conjunctival flap. Bridle traction suture with $4 / 0$ silk was used to expose superior conjunctiva and stabilize the globe. Conjunctival flap was made at superior nasal or superior temporal site. Incision was made in conjunctiva $0.5-1.0 \mathrm{~mm}$ posterior to the limbus with Westcott scissors and Tenon's capsule was carefully dissected from underlying sclera. A vertical relaxing incision was made in conjunctiva in case of tight fibrosed conjunctiva. Wet field cautery was applied at the site of bleeding on sclera. A rectangular flap measuring 3 by $4 \mathrm{~mm}$ with calipers was marked and incised with blade 11. Partial thickness window was created with blade 15 . Piece of trabecular mesh work about $2 \mathrm{~mm}$ by $1 \mathrm{~mm}$ was removed followed by iridectomy with the help of iridectomy scissors. A side port was made to wash anterior chamber and to check the patency of fistula. Anterior chamber was washed with normal saline in case of hyphema and was formed with air. Scleral flap was closed with interrupted 10/0 nylon sutures at two ends. Tenon's and conjunctiva were closed with 10/0 nylon in interrupted fashion. Post operatively topical steroid dexamethasone was prescribed for 8-12 weeks and antibiotic Moxifloxacin for 4 weeks. Steroid antibiotic ointment was given for 2 weeks. Systemic antibiotics (Ciprofloxacin $500 \mathrm{mg}$ BD) were also given. Patients were followed-up to see the IOP, visual acuity and any post-operative complications. Best corrected visual acuity was assessed with the help of Snellen's chart. IOP was measured with Goldman applanation tonometer. Follow-up was done on first postoperative day then on $1^{\text {st }}$ week, $2^{\text {nd }}$ week, $1^{\text {st }}$ month, $3^{\text {rd }}$ month, $6^{\text {th }}$ month and at 1 year. Frequent follow-ups were done if necessary.

Data was analyzed using SPSS version 21. Primary outcome measures were preoperative and postoperative visual acuity, intraocular pressure, number of medications, improvement in visual acuity and failed or successful trabeculectomy. Mean with standard deviation was calculated for numerical 
variables like age, duration of disease, number of preoperative and post-op medications, base line and follow-up IOP. Frequencies were computed for categorical variables like gender, laterality of eye, best corrected visual acuity (BCVA), improvement or deterioration of visual acuity, successful and failed trabeculectomy. One sample $t$ test was used to compare means of IOP and number of medications pre-operatively and post-operatively. $\mathrm{P}$ value of less than 0.05 was taken as significant.

Effectiveness of trabeculectomy was classified into three groups, complete success, qualified success and failed trabeculectomy. Intra ocular pressure of less than $20 \mathrm{~mm} \mathrm{Hg}$ or less at 1 year after trabeculectomy without any medication was labeled as complete success. IOP of $20 \mathrm{~mm} \mathrm{Hg}$ or less with help of medications at 1 year was labeled as qualified success. Trabeculectomy was defined 'fail' in case of IOP of 20 $\mathrm{mm} \mathrm{Hg}$ or more in spite of maximum medical management. ${ }^{13,14}$

\section{RESULTS}

Total number of patients in this study were 52 who underwent trabeculectomy. The detailed demographic features are given in table 1.

Table 1: Demographics of Glaucoma Patients That Underwent Trabeculectomy.

\begin{tabular}{lc}
\hline \multicolumn{2}{c}{ Variables } \\
\hline Mean age & $56.73 \pm 10.9$ SD \\
Min & 33 \\
Max & 74 \\
Male & $36(69.2 \%)$ \\
Female & $16(30.8 \%)$ \\
Duration of glaucoma & $3.8 \pm 2.3$ years \\
Right eye & $32(61.5 \%)$ \\
Left eye & $20(38.5 \%)$ \\
Mean preoperative IOP & $30.96 \pm 6.71$ \\
Improvement in lines & $32(61.5 \%)$ \\
Deterioration in lines & $4(7.7 \%)$ \\
No improvement & $16(30.8 \%)$ \\
\hline
\end{tabular}

Table 2: Preoperative and Postoperative Visual Acuity, Intraocular Pressure and Number of Medications.

\begin{tabular}{llcc}
\hline BCVA & Pre-operative & Post-operative & P value \\
\hline 6/6 to 6/18 & 10 & 10 & \\
6/36 to 6/24 & 10 & 12 & .000 \\
6/60 and less & 24 & 28 & \\
PLPR to LP & 8 & 2 & \\
IOP mm Hg & & & .000 \\
At $3^{\text {rd }}$ month & $30.96 \pm 6.71$ & $14.6 \pm 3.2$ &
\end{tabular}

$\begin{array}{ll}\text { At } 6^{\text {th }} \text { month } & 15.7 \pm 4.0 \\ \text { At } 12^{\text {th }} \text { month } & 15.6 \pm 4.2\end{array}$

No. of

Medications

3.03

1.19

.000

*nonparametric test chi square ^one sample t test, one sample t test

PLPR = perception of light and projection of light,

$\mathrm{LP}=$ light perception

Complete Surgical success was seen in 28 (53.8\%) patients. Qualified success was in 21 (40.4\%) patients requiring additional medication. Failed trabeculectomy was seen in $3(5.8 \%)$ patients requiring surgical intervention.

\section{DISCUSSION}

Trabeculectomy remains the most common non-laser surgical procedure for management of glaucoma. Medical treatment has been found to be insufficient in patients belonging to third world countries because of very poor compliance. ${ }^{15}$ Complete success was seen in $28(53.8 \%)$ patients, qualified success was seen in 21 (40.4\%) patients. Overall success was seen in 49 $(94.2 \%)$ cases. Failed trabeculectomy was seen in 3 (5.8\%) patients at 1 year in this study. A study conducted in Nigerian teaching hospital reported complete success in $34(61.8 \%)$ and qualified success in $15(27.3 \%)$ at 1 year post trabeculectomy. Their overall success was seen in $91.1 \%$. Their failure rate was $6(10.9 \%) .{ }^{14}$ Their mean follow-up time was 3.03 years. Complete success was seen in $66.7 \%$ of Chinese people (18 patients) and qualified success in $16.7 \%$. Their failure rate was seen in $10 \%$ patients at 3 months. ${ }^{16}$ Complete success and qualified success rates of our study are comparable with other studies. ${ }^{14,16}$ Trabeculectomy gradually fails over passage of time due to fibroblastic proliferation and sub conjunctival fibrosis seen as a normal process of wound healing. ${ }^{17}$

Mean pre-operative IOP in our study was $30.96 \pm$ 6.71 and at 1 year it was $15.6 \pm 4.2 \mathrm{~mm} \mathrm{Hg}$. In a Nigerian study pre-operative IOP was $32.5 \pm 6.2 \mathrm{~mm}$ $\mathrm{Hg}$ and 1 year, it was $16.2 \pm 3.7 \mathrm{~mm} \mathrm{Hg} .{ }^{14}$ Tabassam et al had reported pre op IOP $32.7 \pm 12.43$ and at 1 year it was $15.78 \pm 3.71 \mathrm{~mm} \mathrm{Hg} .{ }^{18}$ In all of the above studies mean pre-operative IOP was exceeding $30 \mathrm{~mm}$ $\mathrm{Hg}$ and trabeculectomy had successfully reduced it to $14-16 \mathrm{~mm} \mathrm{Hg}$ at 1 year. The goal of glaucoma management is to achieve a target pressure, which will 
help to preserve visual function. ${ }^{19}$ Target pressure is different for every patient and it has to be reassessed according to visual field progression and optic nerve head changes for each patient. If IOP remains high normal after trabeculectomy, patient can end up with legal blindness. Fluctuation of IOP will lead to optic disc damage and visual field progression. A surgeon has achieved his goal if trabeculectomy is attaining and maintaining target pressure for his patient.

In this study, there was no change in visual acuity in $30.8 \%$ of patients after trabeculectomy. Reduction in visual acuity was seen in $7.7 \%$ of patients. Study by Tabassam et al documented no change in visual acuity at 1 year. ${ }^{18}$ There was no difference in visual acuity in a study done in Nigerian teaching hospital and in Chinese population. ${ }^{14,16}$ However, they did not specify any improvement in lines. ${ }^{14,18}$ It has been reported that filtration surgery may lead to decrease quality of vision in some patients. However, these changes are reversible and visual acuity returns to pre-operative levels within three-month period. Trabeculectomy may induce some refractive changes like changes in corneal contour, anterior chamber depth and axial length..$^{20,21}$ Every single line on Snellen's chart is important for glaucoma patient who realizes that with passage of time it will deteriorate. If trabeculectomy stabilizes and maintains visual acuity of POAG patient, it will certainly have effects on quality of life.

Decrease in number of anti-glaucoma medications after trabeculectomy from 3.5 to 1.5 drops was also reported in a study conducted in Sweden. ${ }^{20}$ Trabeculectomy has an advantage of reducing patient's dependence on medication. ${ }^{8}$ Patients on topical anti-glaucoma medications have increased incidence of ocular surface diseases. ${ }^{22}$ Trabeculectomy maintains quality of life by lowering number of topical medications. ${ }^{20,22}$ and quality of vision affected by use of topical medications with preservatives.

Males were twice than females with ratio of 2.2:1 in our study. Other studies also demonstrated male preponderance. ${ }^{13,14,17,18} \mathrm{We}$ did not use antimetabolites in routine cases. They are associated with frequent wound leaks, late bleb leaks, hypotony so they are advisable in young and Negro patients with high risk of postoperative fibrosis. ${ }^{23}$

Glaucoma is a disease that cannot be cured but its progression can be controlled successfully. Glaucoma is neither treatable nor preventable but appropriate management can halt its progression. ${ }^{24}$ Not only surgical skill but appropriate postoperative bleb management is crucial for a successful surgery.

Limitation of our study is being a retrospective design and a limited sample size. Visual field progression analysis and documenting optic nerve head changes could have made our study results more comprehensive.

\section{CONCLUSION}

Conventional trabeculectomy is effective in lowering IOP in primary open angle glaucoma patients. It maintains IOP within normal range with and without anti-glaucoma medications in most of the patients at 1 year follow-up.

\section{Ethical Approval}

The study was approved by the Institutional review board/Ethical review board.

\section{Conflict of Interest}

Authors declared no conflict of interest.

\section{REFERENCES}

1. Quigley HA. Number of people with glaucoma worldwide. Br J Ophthalmol. 1996; 80 (5): 389-93.

2. Resnikoff S, Pascolini D, Mariottia SP. Global magnitude of visual impairment caused by uncorrected refractive errors in 2004. Bull World Health Organ, 2008; 86: 63-70.

3. Jacob A, Thomas R, Koshi SP, Braganza A, Muliyil J. Prevalence of primary glaucoma in an urban south Indian population. Indian J Ophthalmol. 1998; 46 (2): 81.

4. Thapa SS, Paudyal I, Khanal S, Twyana SN, Paudyal G, Gurung R, et al. A population-based survey of the prevalence and types of glaucoma in Nepal: the Bhaktapur Glaucoma Study. Ophthalmology, 2012; 119 (4): 759-764.

5. Khan L, Ali M, Qasim M, Jabeen F, Hussain B. Molecular basis of glaucoma and its therapeutical analysis in Pakistan: an overview. Biomed Res Ther. 2017; 23; 4 (03): 1210-1227.

6. Radhakrishnan S, Quigley HA, Jampel HD, Friedman DS, Ahmad SI, Congdon NG, et al. Outcomes of surgical bleb revision for complications of trabeculectomy. Ophthalmology, 2009; 116 (9): 17131718 . 
7. Lichter PR, Musch DC, Gillespie BW, Guire KE, Janz NK, Wren PA, et al. CIGTS Study Group. Interim clinical outcomes in the Collaborative Initial Glaucoma Treatment Study comparing initial treatment randomized to medications or surgery. Ophthalmology, 2001; 108 (11): 1943-1953.

8. Landers J, Martin K, Sarkies N, Bourne R, Watson P. A twenty-year follow-up study of trabeculectomy: risk factors and outcomes. Ophthalmology, 2012; 119 (4): 694-702.

9. Dada T, Kusumesh R, Bali SJ, Sharma S, Sobti A, Arora V, et al. Trabeculectomy with combined use of subconjunctival collagen implant and low-dose mitomycin C. J Glaucoma. 2013; 22: 659-662.

10. The Fluorouracil Filtering Surgery Study Group. Fiveyear follow-up of the Fluorouracil Filtering Surgery Study. Am J Ophthalmol. 1996; 121: 349-366.

11. Singh K, Mehta K, Shaikh NM, Tsai JC, Moster MR, Budenz DL, et al. Trabeculectomy with intraoperative mitomycin $\mathrm{C}$ versus 5-fluorouracil: prospective randomized clinical trial. Ophthalmology, 2000; 107 (12): 2305-2309.

12. Schwartz K, Budenz D. Current management of glaucoma. Current Opinion in Ophthalmology, 2004; 15 (2): 119-126.

13. Agbeja-Bayeroju AM, Omoruyi M, Owoaje ET. Effectiveness of trabeculectomy on glaucoma patients in Ibadan. Afr J Med Sci. 2001; 31: 39-44

14. Adegbehingbe BO, Majemgbasan T. A review of trabeculectomies at a Nigerian teaching hospital. Ghana Med J. 2007; 41 (4).

15. Nouri-Mahdavi K, Hoffman D, Coleman AL, Liu G, Li G, Gaasterland D, et al. Predictive factors for glaucomatous visual field progression in the Advanced Glaucoma Intervention Study. Ophthalmology, 2004; 111 (9): 1627-1635.

16. Choy BN. Comparison of surgical outcome of trabeculectomy and phacotrabeculectomy in Chinese glaucoma patients. Intern J Ophthalmol. 2017; 10 (12): 1928.

17. Agbeja-Bayeroju Am, Owoaje ET, Omoruyi M. Trabeculectomy in young Nigerian patients. Afr J Med Sci. 2002; 31 (1): 33-35.
18. Tabassum G, Ghayoor I, Ahmed R. The effectiveness of conventional trabeculectomy in controlling intraocular pressure in our population. Pak J Ophthalmol. 2013; 29 (1): 26-30.

19. Rao HL, Addepalli UK, Jonnadula GB, Kumbar T, Senthil S, Garudadri CS. Relationship between intraocular pressure and rate of visual field progression in treated glaucoma. J Glaucoma. 2013; 22: 719-724.

20. Binibrahim IH, Bergström AK. The role of trabeculectomy in enhancing glaucoma patient's quality of life. Oman Journal of Ophthalmology, 2017; 10 (3): 150.

21. Wagschal LD, Trope GE, Jinapriya D, Jin YP, Buys YM. Prospective randomized study comparing ExPRESS to trabeculectomy: 1-year results. J Glaucoma, 2015; 24 (8): 624-629.

22. Hirooka $\mathbf{K}$, Nitta $\mathbf{E}$, Ukegawa $\mathbf{K}$, Tsujikawa $A$. Vision-related quality of life following glaucoma filtration surgery. BMC Ophthalmology, 2017; 17 (1): 66.

23. Butt NH, Ayub MH, Ali MH. Challenges in the management of glaucoma in developing countries. Taiwan J Ophthalmol. 2016; 6 (3): 119-122.

24. Robin A, Grover DS. Compliance and adherence in glaucoma management. Indian J Ophthalmol. 2011; 59 (Suppl. 1): S93.

\section{Authors' Designation and Contribution}

Erum Shahid; Assistant Professor: Concepts, Design, Literature research, Data acquisition, Data analysis, Statistical analysis, Manuscript preparation, Manuscript editing, Manuscript review.

Uzma Fasih; Associate Professor: Concepts, Design, Data acquisition, Manuscript editing, Manuscript review.

Arshad Shaikh; Professor \& Head of Department: Concepts, Design, Data analysis, Manuscript editing, Manuscript review. 